\title{
Observational Aspect of Black Hole Dark Matter
}

\author{
L. Stodolsky, \\ Max-Planck-Institut für Physik (Werner-Heisenberg-Institut) \\ Föhringer Ring 6, 80805 München, Germany
}

March 8, 2021

\begin{abstract}
Advances in high angular resolution astronomy make it conceivable that black hole dark matter could be detected via angular deviation effects.

Assuming the dark matter in the galaxy is made of solar mass black holes, there is a non-trivial probability that a line-of-sight through the galaxy, leads to $\mu$ arcsec 's deviations, a value that has been discussed for various astronomical projects.

In cosmology the effects are magnified by an increased density at early times and an opening of angles due to redshift. We stress the interest of the resolution in observations on the $\mathrm{CMB}$ and emphasize the distinction between this and the power spectrum. If the dark matter is made of primordial black holes, present at the CMB, random deflections of the CMB photons lead to a limit on the angular resolution, approximately $3 \times 10^{-7} \sqrt{M / M_{\odot}} \mathrm{rad}$, with $M$ the mass of the black holes. Using the resolutions of $\sim 10^{-3} \mathrm{rad}$ demonstrated in observations of the "acoustic peaks " then implies the limit $\left(M / M_{\odot}\right) \lesssim 10^{7}$. While this large value seems uninteresting, improved resolutions would lead to significant exclusion limits or conceivably the discovery of primordial black holes.
\end{abstract}

\section{Introduction}

The problem of the nature of the dark matter is and remains one of the primary and most fascinating questions of contemporary science. While the search for elementary particle dark matter, particularly by means of the cryogenic technique 1, seems at present the most plausible path to its elucidation, some other possibilities have been discussed.

One of the most interesting of these possibilities is that the dark matter is made of black holes, primordial objects presumably originating from the very early universe. These have not been definitively excluded and it has been suggested that they could help in the understanding of structure formation in cosmology [2].

Discussions concerning this possibility have necessarily relied on indirect arguments. But it certainly would be more satisfying if there were direct observational evidence, for or against the black hole hypothesis. Here we would like 
to consider how it may be possible to obtain such evidence by means of high angular resolution observations.

For a preliminary orientation we first consider the situation for the galaxy, and then turn to the CMB (Cosmic Microwave Background ), where general relativistic effects lead to large enhancements.

\section{Deflection and Distance}

Our arguments are based on the deflection of light by massive bodies, as in the famous bending of light by the sun. If black holes make up the dark matter, the interstellar or intergalactic medium would be very "lumpy" on short distance scales, as opposed to dark matter made of elementary particles.

A standard result in General Relativity 3 is that the deflection angle $\delta \alpha$ of a ray passing a gravitating object of mass $\mathrm{M}$ at impact parameter $b$, is

$$
\delta \alpha=\frac{4 G M}{c^{2} b}=2 \frac{r_{s}}{b}=\frac{6.0 \mathrm{~km}}{b}\left(M / M_{\odot}\right)=\frac{6.3 \times 10^{-13} \mathrm{ly}}{b}\left(M / M_{\odot}\right),
$$

where we express $M$ in terms of the Schwarzschild radius $r_{s}=2 G M$ and normalize to the mass of the sun, $M_{\odot}$, where one has $r_{s}=3.0 \mathrm{~km}$. Here and in the following we use $c=1$ units.

Thus for a deflection of $\delta \alpha$ or more there is a certain impact paramter $b$, to which we can associate a " cross section"

$$
\begin{aligned}
\pi b^{2}=\pi\left(\frac{2 r_{s}}{\delta \alpha}\right)^{2} & =1.1 \times 10^{2}\left(\frac{1}{\delta \alpha}\right)^{2}\left(M / M_{\odot}\right)^{2} k m^{2} \\
& =1.3 \times 10^{-24}\left(\frac{1}{\delta \alpha}\right)^{2}\left(M / M_{\odot}\right)^{2} l y^{2}
\end{aligned}
$$

This is the cross section for having, in a single passage near a massive object, a deflection $\delta \alpha$ or more.

(Here and in the following we speak of the black holes as having a single, unique mass; if instead there is a spectrum of masses, $M$ should be understood as the average mass. )

If we consider $\delta \alpha$ 's on the order of a $\mu$ arcsec $=4.8 \times 10^{-12} \mathrm{rad}$, as in long baseline interferometry [4, this cross section can be $\sim l y^{2}$ for a solar mass object. Equivalently, passages at distances $\sim l y$ are potentially observable given such resolutions.

\section{$3 \quad$ Probability of a $\delta \alpha$}

One may turn Eq2 2 into the probability for a deflection $\delta \alpha$ (or more) for a photon traveling a certain path by multiplying by the column density

$$
\operatorname{Prob}_{\delta \alpha}=\text { cross section } \times \text { column density }=\pi\left(\frac{2 r_{s}}{\delta \alpha}\right)^{2} \times \rho_{2},
$$


where the column density $\rho_{2}$ is the two-dimensional density of the total number of black holes along the line-of-flight, projected on the perpendicular plane. (We are taking $\operatorname{Prob}_{\delta \alpha}$ to be small, a value approaching one implies multiple encounters. )

The $\rho_{2}$ can in turn be expressed in terms of the presumably approximately known dark matter mass column density and the unknown black hole mass $M$ as

$$
\rho_{2}=\mu / M,
$$

where the dark matter mass column density is expressed as $\mu /$ unit area, with $\mu$ a mass.

Since $r_{s}^{2} \sim M^{2}$ one sees that $\mathrm{Eq} 3$ is proportional to $M$ and vanishes as $M \rightarrow 0$. We are working with a constant, given, dark matter mass density, so in the limit $M \rightarrow 0$ the medium becomes perfectly smooth. This is in accord with the expectation that the deflection effect depends on how "lumpy" the medium is and would be expected to vanish, say, when the dark matter consists of a smooth "gas" of elementary particles .

\section{Galaxy}

We first apply the above estimates to the case of the galaxy. In his original discussion of 'Machos'(Massive compact halo objects), Paczynski [5] briefly mentioned this possibility of the direct observation of angular deviations, but dismissed it as immeasurably small. However, since that time there have been great improvements in angular resolutions; also a brief discussion for the galaxy will allow us to establish the ideas without the complications of general relativity.

While an accurate calculation of $\rho_{2}$ requires an integration of the dark matter density along the flight path, we can crudely estimate it for a source in the Milky Way (or for rays traveling through a nearby galaxy ) by taking the presumed dark matter density near the earth of $0.4 \mathrm{GeV} / \mathrm{cm}^{3}=7.1 \times 10^{-28} \mathrm{~kg} / \mathrm{cm}^{3}=$ $6.0 \times 10^{26} \mathrm{~kg} / \mathrm{ly}{ }^{3}[9]$ and a typical galactic travel distance of $10^{4}$ light years. One thus obtains

$$
\rho_{2} \approx \frac{1}{M}\left(6.0 \times 10^{26} \mathrm{~kg} / \mathrm{ly} y^{3}\right) \times 10^{4} \mathrm{ly}=3.0 \times\left(M_{\odot} / M\right) / l y^{2} \quad \text { galaxy }
$$

Combining $\mathrm{Eq} 5$ and $\mathrm{Eq} 3$ one has the probability of a deflection of $\delta \alpha$ or more in a typical passage through the galaxy

$\operatorname{Prob}_{\delta \alpha} \approx 3.9 \times 10^{-24}(1 / \delta \alpha)^{2}\left(M / M_{\odot}\right)=1.6 \times 10^{-1} \times\left(1 / \delta \alpha^{\prime}\right)^{2}\left(M / M_{\odot}\right) \quad$ galaxy.

In the first writing $\delta \alpha$ is in radians, while in the second $\delta \alpha^{\prime}$ is in $\mu a r c s e c$. Thus if the dark matter is made up of solar mass (or more) black holes, a ray crossing the galaxy has a substantial chance of a several $\mu$ arcsec deflection. This estimate is of course quite approximate, and will depend on whether the flight path is through regions of high or low dark matter density. 
The next question is how this very small angular deflection might be detected, given that there are generally larger effects from the motion of the source and the earth. To obtain an observable signal, one might look for a motion of $\delta \alpha$. The black hole, like other objects in the galaxy, will be moving with some velocity. This velocity will lead to a change in $b$ in Eq1 and so a change in $\delta \alpha$. Since we are considering passages at distances $b$ on the order of a $l y$ or less and the typical velocity of objects in the galaxy $v \approx 2 \times 10^{-3}$ corresponds to a distance traveled $\approx 2 \times 10^{-3} l y$ in one year, it is possible that a significant change in $b$ can occur over a timescale of years. The presence of the black holes would be thus signaled as a small 'noise', with a timescale on the order of years, on the more smoothly varying angular positions of the sources.

A shorter timescale for the 'noise' could be obtained by raising the threshold of detection for $\delta \alpha$, implying smaller $b$, but then a reduced probability $\operatorname{Prob}_{\delta \alpha}$. This behavior with respect to threshold would be useful in establishing the reality of a possible posit;ive signal.

\section{CMB As Source}

We now turn to the CMB, the most remote soure imaginable. This turns out to be of fundamental interest since, as we shall see, it is in principle possible, although observationally difficult, to set significant limits, or perhaps to discover primrodial black holes through observations on the CMB.

While there have been direct observations concerning black holes, these observations have been in the nearby universe, as in [4, or through the observation of accretion disks, or by rapid motions of stars in the center of the Milky Way 7 . On the other hand, arguments concerning primordial black holes have mainly revolved around their effects on cosmic evolution as in 2]. In contrast, our method would amount to a direct observation.

Very briefly our point is, as familiar in particle or atomic physics, that scattering centers between a source of radiation and a detector or observer lead to

a loss of angular resolution, We apply this point to the presence of black holes between us and the CMB. Thus instead of considering a particular object as the source, we examine the typical deflection of a photon from the CMB and argue that, due to the randomness of this deflection, smaller angular features will be washed out. That is, the presence of primordial black holes implies a limit on the possible angular resolution in CMB observations.

An important aspect of the calculation, as seen below, is that the effect largely arisess from deflections at early times, when the cosmological scale factor $a(t)$ is small. Without this enhancement the effect would be undetectably small. Hence a positive observation would strongly suggest that the deflecting objects are primordial.

This point concerning observations on the CMB distinguishes primordial black holes from other compact gravitating object that might form in the course of cosmic evolution, but not present at the CMB, such as "Machos", "Brown Dwarfs", and so forth. With the present understanding of cosmology, such 
compact objects would exist after the formation of structure, and would not be present at the CMB. On the other hand, primordial black holes would presumably arise from very early times [2].

The perhaps surprising conclusion that angular deviations might be observable results from some general relativistic effects in cosmology, namely an opening of the deflection angle seen by the observer, and the high density of dark matter at earlier times. These lead to a large quantitative enhancement relative to Eq[6. As said, the enhancement is large and occurs at early times or high redshift and distinguishes the primordial black holes effect from those originating from density concentrations developing out of a smooth background. The later will necessarily lead to much smaller effects since there is not the enhancement at early times.

\subsection{Opening of Angles}

A deflection angle produced at high redshift is magnified when observed "now", due to the cosmological redshift. We work in the simplest FRW model for cosmology, where one has the scale factor $a(t)=\left(t / t_{\text {now }}\right)^{2 / 3}$ after the formation of the $\mathrm{CMB}$, with $t_{\text {now }}=2.9 \times 10^{17} \mathrm{~s}$. (Note this notation introduces a factor $3 / 2$ between $t_{\text {now }}$ and the hubble parameter. )

In the local frame of the black hole, the photon before deflection has the 4vector $k$ and after deflection $k^{\prime}$. The scalar 4-product is $k k^{\prime}=\omega^{2}(1-\cos \delta \alpha) \approx$ $\frac{1}{2} \omega^{2}(\delta \alpha)^{2}$. We take the two photons to have the same frequency and to differ by a small angle, as for photons deflected by a massive black hole. A scalar product is conserved under parallel transport, and since the photons come to us by free fall or parallel transport, a deflection $\delta \alpha_{t}$ at cosmological time $t$ has the relation to the $\delta \alpha$ at the time 'now', $\left(\omega_{t} \delta \alpha_{t}\right)^{2}=\left(\omega_{\text {now }} \delta \alpha\right)^{2}$.

Therefore the deviation angles are in the ratio of the frequencies and so are increased by the redshift.

$$
\delta \alpha=\frac{1}{a(t)} \times \delta \alpha_{t}
$$

where $a(t)$ is the scale factor at the cosmological time $t$ of the scattering.

This means that a deflection $\delta \alpha$ 'now' originates from a smaller deflection at high redshift, and since these will occur at larger impact parameter, they have a higher probability than would be the case without this angular effect. (This logic can also be used to show that a nonrelativistic transverse motion has only a small effect on $\delta \alpha$; also applicable for the galaxy case.)

\subsection{Increase of Density}

A second effect is the increased density of the dark matter at early times, which is expected to vary as $1 / a^{3}$, such that number density at time $t$ is $\rho / a^{3}$, where $\rho$ is the present number density. The contribution to the column density from a cosmic time interval $d t$ is then 


$$
d \rho_{2}=\rho \frac{1}{a^{3}} d t=\frac{\mu}{M} \frac{1}{a^{3}} d t,
$$

where $\mu$ is the cosmological dark matter mass density at present [6].

\subsection{Probability Integral}

Taking these two effects into account

$$
d \operatorname{Prob}_{\delta \alpha}=\pi b^{2}(t) d \rho_{2}=\pi\left(\frac{2 r_{s}}{(a(t) \delta \alpha}\right)^{2} d \rho_{2},
$$

using Eq[7 and Eq[ to give $b(t)=2 r_{s} /(a(t) \delta \alpha)$. Combining with $\mathrm{Eq} 8$

$$
\int d \operatorname{Prob}_{\delta \alpha}=\frac{\mu}{M} \pi\left(\frac{2 r_{s}}{\delta \alpha}\right)^{2} \int \frac{1}{a^{5}} d t,
$$

The factor $1 / a^{5}$ will lead to large enhancement over the simple dimensional factors:

$$
\int_{c m b}^{\text {now }} d t \frac{1}{a^{5}}=t_{\text {now }} \frac{3}{7}\left(x^{7 / 3}-1\right)=7.2 \times 10^{9} \times t_{\text {now }}
$$

with $x=t_{\text {now }} / t_{c m b}=2.4 \times 10^{4}$, integrating over the time since $t_{c m b}=3.8 \times 10^{5} \mathrm{y}$.

We are left with the task of evaluating the dimensional factor

$$
\frac{\mu}{M} \pi\left(\frac{2 r_{s}}{\delta \alpha}\right)^{2} t_{n o w}
$$

The "cross section" was evaluated in Eq2

$$
\pi\left(\frac{2 r_{s}}{\delta \alpha}\right)^{2}=1.3 \times 10^{-24}\left(\frac{1}{\delta \alpha}\right)^{2}\left(M / M_{\odot}\right)^{2} l y^{2}
$$

The remaining factor $\frac{\mu}{M} t_{\text {now }}$ can be interpreted as the column density for a distance $t_{\text {now }}(\sim$ Hubble distance) at the present dark matter density, without the general relativistic effects. To evaluate this we take the present dark matter mass density $\mu$ at $1 / 4$ the critical value [9]:

$$
\mu=1.0 \times 10^{-9} M_{\odot} / l y^{3},
$$

which together with $t_{\text {now }}=9.2 \times 10^{9} \mathrm{yr}$ yields

$$
\frac{\mu}{M} t_{n o w}=9.2 \times\left(M_{\odot} / M\right) / l y^{2}
$$

Interestingly, this is about the same as the estimate for the galaxy in Eq[5 a factor $10^{6}$ in the densities has been cancelled by a similar factor in the distances.

Thus the main difference vis-a-vis the galactic effects arises from the large factor of Eq11, from the 1/ $a^{5}$ behavior. The dominant effect thus comes from small $a$. The necessary "clumpiness", noted at the end of section 3 , originates from the primordial black holes present at early times. This is quite different 
from accretion or gravitational accumulation mechanisms starting from an originally smooth medium and gradually building up at much later times. Such late-time buildup of density contrasts would not benefit greatly from the small $a$ magnification and should lead to much smaller results than those given here. The primordial black holes, if they exist, would naturally also be expected to grow by accretion, but, as said, the dominant effect will come from their size at around the time of the formation of the CMB.

To finally estimate the integrated $\mathrm{Eq} 10$ for photons from the CMB we put together Eq[11] Eq13, and Eq[15, to find

$$
\operatorname{Prob}_{\delta \alpha}=8.6 \times 10^{-14}\left(\frac{1}{\delta \alpha}\right)^{2}\left(M / M_{\odot}\right)=3.7 \times 10^{9}\left(\frac{1}{\delta \alpha^{\prime}}\right)^{2}\left(M / M_{\odot}\right)
$$

where again $\delta \alpha$ is in radians while $\delta \alpha^{\prime}$ is in $\mu$ arcsec .

\subsection{Discussion}

To convert these results into a possibly observable effect, consider the question of the angular resolution possible in observations on the CMB. When $\operatorname{Prob}_{\delta \alpha}$ is of order one, a photon from a point on the CMB, will undergo a deflection $\delta \alpha$ with high probability. Since these deflections are random in direction, the angular position of points will be altered by $\delta \alpha$. This implies a limit on the angular resolution, set by the requirement that $\operatorname{Prob}_{\delta \alpha}$ be less than one. Setting $\operatorname{Prob}_{\delta \alpha} \approx 1$ in Eq16 gives

$$
\delta \alpha_{l i m} \approx 2.9 \times 10^{-7} \sqrt{\left(M / M_{\odot}\right)} \quad \delta \alpha_{l i m}^{\prime} \approx 6.1 \times 10^{4} \sqrt{\left(M / M_{\odot}\right)},
$$

as an approximate limit for the best obtainable resolution in observations on the $\mathrm{CMB}$, in radians or $\mu$ arcsec respectively.

To see how this might work in practice, we take the observation of the "acoustic peaks" in the temperature fluctuations of the CMB. (We stress that here we are not interested in the temperature fluctuations themselves but simply in the demonstration of an angular resolution.) These features have been observed out to $l \sim 1000$, implying angular resolutions of about $\sim 10^{-3} \mathrm{rad}$. Using Eq[17 this implies an approximate upper limit on the mass of possible black holes

$$
\left(M / M_{\odot}\right) \lesssim 10^{7}
$$

The examples of very massive black holes found up to the present time are in the range of millions of solar masses as in [4] [7, and the existence of even larger objects should have been evident. For a discussion of such limits see [10.

Thus Eq18 does not seem a very stringent limit, However, the argument does suggest that higher resolution observations on the CMB could lead to significant restrictions or perhaps even positive evidence for dark matter black holes. For example, if a resolution in the $10 \mu$ arcsec range as in 4 were possible, then from the second formula of $\mathrm{Eq} 17$ one is in the $\left(M / M_{\odot}\right) \sim 10^{-7}$ regime, which would be quite interesting. In [4] earth-size baselines were used, so it seems 
that for the present purposes achievement of such spectacular resolutions would imply extraterrestial arrangements 4$]$.

A positive discovery of primordial black holes as compared to merely setting upper limits is of course the more difficult task, since one must eliminate other possible angular averaging effects, both instrumental and natural. In this connection it should be noted that Eq17 is frequency independent, reflecting the achromatic behavior of light in gravitational fields, a feature that would not be expected for many background effects.

We should stress that our limits, as in Eq[18, are arrived at by assuming that the dark matter is made of, or largely made of, black holes present at the formation of the CMB; and so we can say nothing about the present existence of other objects like 'stupendous' black holes, perhaps arising in the course of cosmic evolution [12.

Finally we note, that although we speak about black holes, the arguments evidently would apply to any massive, compact gravitating objects making up the dark matter and present around the time of the formation of the CMB. The objects might even be relatively extended, our estimates should apply as long as their effective cross section doesn't become comparable with that of Eq2.

\subsection{Power Spectrum vs. Angular Resolution}

The power spectrum, or equivalently directional anisotropies, of the CMB has been a much discussed subject, with a large literature [11, To avoid possible confusion, we would like to stress that the resolution on the CMB, which we have introduced here, is not the same thing. We contrast the two: the power spectrum of the $\mathrm{CMB}$ on the one hand, and the angular resolution in observing the $\mathrm{CMB}$ on the other.

That the two cannot be the same is immediately evident when one recognizes that the two convey different sorts of information. The power spectrum gives information on the nature of the CMB itself, reflecting primordial density fluctuations. On the other hand, the angular resolution provides information on the 'medium' between us and the CMB. For the angular resolution question, the role of the CMB is simply to provide a 'source' at high redshift.

Of course, the existence of a finite resolution affects analysis of the power spectrum. A finite resolution washes out structure as small angles and leads to a softening of the power spectrum at small angles or high $l$. Indeed, once the resolution is well understood it can be deconvolved from the data to obtain the true power spectrum.

The difference between the two is also made clear by considering how they are determined. To determine a power spectrum, one takes the fourier transform of a two-point correlation function. The two-point correlation function, in turn, is an average over the data-in this case points or pixels on the sky. The angular resolution on the other hand, concerns, in principle, how well a single point can be defined. No averaging is implied, and it could theoretically be determined from one point on the sky, if fine enough features existed. 


\subsection{Open question}

In connection with this last remark, it is likely that are no very small angle features on the CMB, analogous to the "acoustic peaks", which could be used to demonstrate the desired high angular resolution. A way around this problemto determine a resolution when there are no suitable small targets-might be addressed by examining the coherence of the radiation field over long baselines. This is under study 13$]$.

\section{References}

[1] A. Drukier and L. Stodolsky, Phys. Rev. D 30, 2295 (1984). doi:10.1103/PhysRevD.30.2295; M. W. Goodman and E. Witten, Phys. Rev. D 31, 3059 (1985). doi:10.1103/PhysRevD.31.3059. For recents results see A. H. Abdelhameed et al. [CRESST Collaboration], arXiv:1904.00498 [astro-ph.CO], Phys. Rev. D 100, 102002 (2019). A general introduction is in L. Stodolsky, Phys. Today 44N8, 24 (1991), doi:10.1063/1.881266.

[2] B. Carr and J. Silk, Mon. Not. Roy. Astron. Soc. 478, no. 3, 3756 (2018) doi:10.1093/mnras/sty1204 [arXiv:1801.00672 [astro-ph.CO]]. See also B. J. Carr, Astrophys. J. 201, 1 (1975), doi:10.1086/153853, P. H. Frampton, Mod. Phys. Lett. A 33, no. 31, 1850176 (2018) doi:10.1142/S0217732318501766 arXiv:1802.05678 [gr-qc]], B. Carr, S. Clesse, J. Garcia-Bellido and F. Kuhnel, arXiv:1906.08217 Phys. Dark Univ. 3110075 (2021).

[3] See for example Eq. 8.5.8 in Gravitation and Cosmology by S. Weinberg, John Wiley and Sons, (1972). For a brief explanation see Wikipedia Gravitational Lens, end of section "Explanation in terms of space-time curvature".

[4] The EHT project, K. Akiyama et al. [Event Horizon Telescope Collaboration], Astrophys. J. 875, no. 1, L1 (2019) doi:10.3847/2041-8213/ab0ec7 [arXiv:1906.11238 [astro-ph.GA]], using earth-sized baselines, reached resolutions in the several $\mu$ arcsec range. K. G. Carpenter et al discuss resolutions in the 0.1 milli-arcsec range, arXiv:1908.05665. The Space Interferometry Mission (Wikipedia) aims at such resolutions, in the optical range.

[5] B. Paczynski, Astrophys. J. 304, 1 (1986). doi:10.1086/164140. For limits in the solar mass range see R. A. Allsman et al. [Macho Collaboration], Astrophys. J. 550, L169 (2001) doi:10.1086/319636 astro-ph/0011506.

[6] The principle of calculation we use is similar to that for other effects such as microlensing. See for example Eq 15 of E. Zackrisson and T. Riehm, Astronomy and Astrophysiscs 275, 453, (2007), arXiv:0709.1571 However, it should be emphasized that there 'high redshift' is meant a $z$ of 5 or 10 , 
while in our discussion concerning the CMB we deal with $z$ in the thousands. Thus the issues discussed in this reference are not directly significant for our cosmological considerations, which are dominated by the highest $z$.

[7] See "A Star in a 15.2 year orbit around the super massive black hole at the center of the Milky Way", R. Schodel, T. Ott, R. Genzel et al. Nature 419, 694, (2002).

[8] Multipoles up to $l \sim 10^{3}$ are discussed in N. Aghanim et al. [Planck Collaboration], arXiv:1807.06207 [astro-ph.CO]. Astr.Astrphys. 641 A3 (2020).

[9] We take all parameters from the Particle Data Group booklet 2016, extracted from C. Patrignani et al. [Particle Data Group], "Review of Particle Physics," Chin. Phys. C 40, no. 10, 100001 (2016). doi:10.1088/1674$1137 / 40 / 10 / 100001$

[10] A discussion of maximum masses for black holes is found in A. D. Dolgov and S. Porey, arXiv:1905.10972 [astro-ph.CO].

[11] W. Hu and N. Sugiyama, "Toward understanding CMB anisotropies and their implications," Phys. Rev. D 51, 2599-2630 (1995) doi:10.1103/PhysRevD.51.2599 arXiv:astro-ph/9411008 [astro-ph]].

[12] B. Carr, F. Kuhnel, and L. Vishnelli, arXiv 2008.08077 discuss such possibilities.

[13] L. Stodolsky unpublished. 